\title{
Agminated Nodules on the Scalp
}

\author{
Lan Zhang, MD; Yang Yang, MD; Song Zheng, MD
}

\section{Eligible for 1 MOC SA Credit From the ABD}

This Photo Challenge in our print edition is eligible for 1 self-assessment credit for Maintenance of Certification from the American Board of Dermatology (ABD). After completing this activity, diplomates can visit the ABD website (http://www.abderm.org) to self-report the credits under the activity title "Cutis Photo Challenge." You may report the credit after each activity is completed or after accumulating multiple credits.
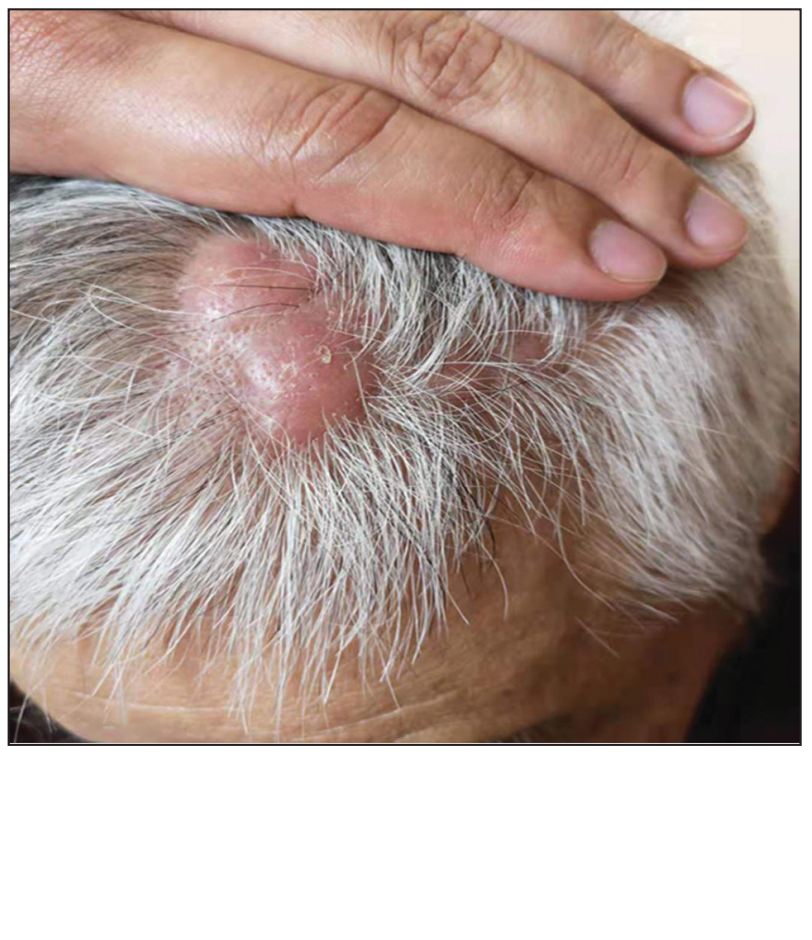

A 67-year-old man presented with pink nodules on the scalp that were enlarging and increasing over the course of 2 months. The patient was otherwise healthy, had no constitutional symptoms such as fever or weight loss, and did not note pruritus or pain. His medications included telmisartan and Salvia miltiorrhiza for hypertension and coronary heart disease, respectively. He had been a heavy smoker for 44 years. Physical examination revealed several dome-shaped, pink nodules with smooth surfaces distributed in an agminated appearance on the scalp. The lesions were indurated and ranged from 1 to $5 \mathrm{~cm}$ in diameter.

\section{WHAT'S YOUR DIAGNOSIS?}
a. atypical fibroxanthoma
b. cutaneous angiosarcoma
c. cutaneous follicle center lymphoma
d. cutaneous metastatic carcinoma
e. cutaneous pseudolymphoma

From the Department of Dermatology and Key Laboratory of Immunodermatology, First Hospital of China Medical University, Shenyan. The authors report no conflict of interest.

This work was supported by grants from the National Natural Science Foundation of China (81803148) and the National Key Research and Development Program of China (2016YFC0901504).

Correspondence: Song Zheng, MD, First Hospital of China Medical University, Nanjing N St, Heping District, Shenyang 110001, China (zhengsongcmu@163.com).

doi:10.12788/cutis.0294 


\section{THE DIAGNOSIS:}

\section{Cutaneous Angiosarcoma}

马 iopsy revealed a cellular neoplasm consisting of atypical polygonal cells with a hobnailed appearance, vasoformative characteristics, and rare extravasated erythrocytes. The tumor had an infiltrative growth pattern as demonstrated by dissecting dermal collagen and a poorly defined border with adjacent normal tissue (Figure 1). Immunohistochemistry revealed that the lesion was positive for CD31 and D2-40 (Figure 2) but negative
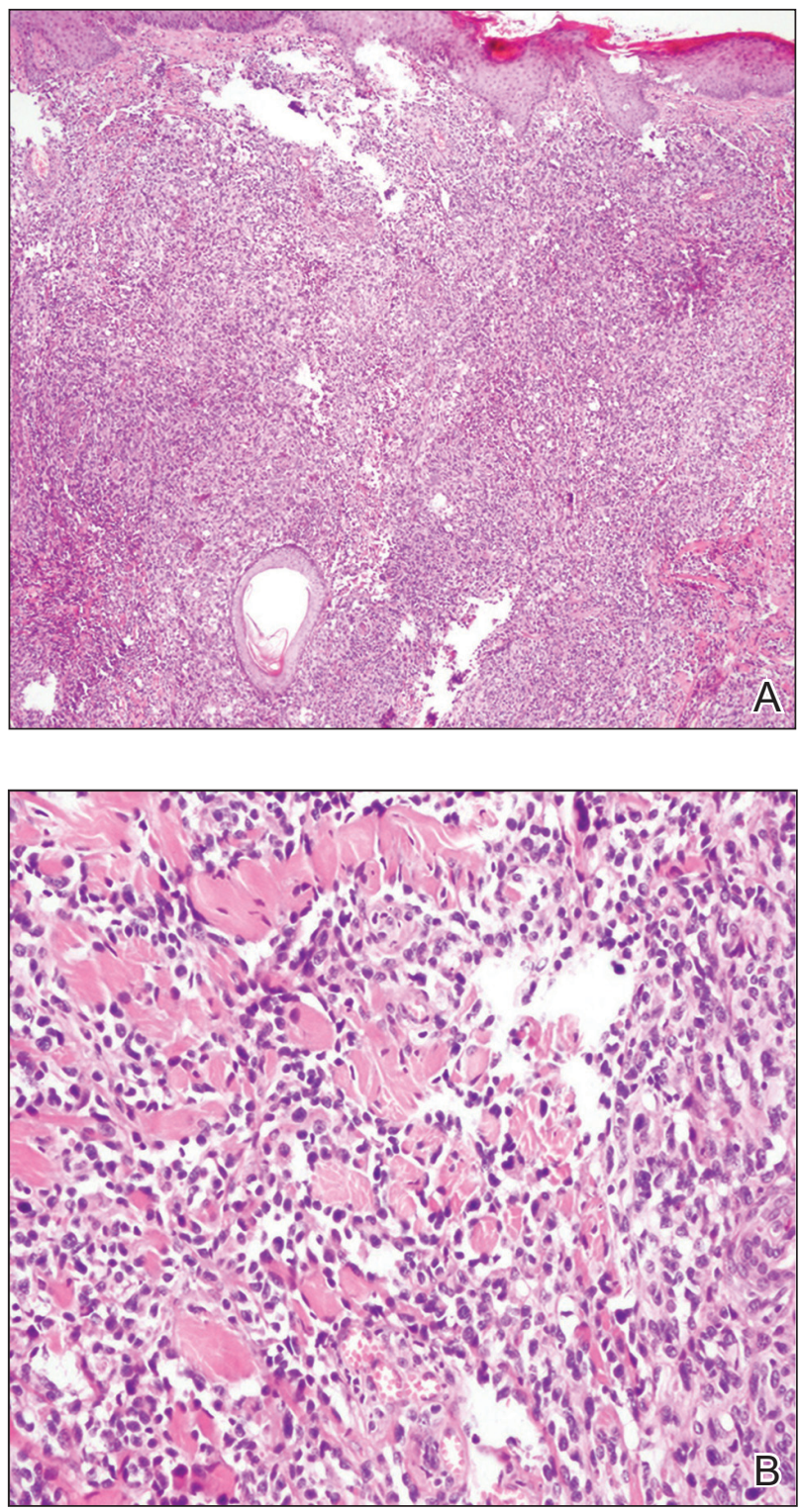

FIGURE 1. A and B, Histologic examination revealed a cellular neoplasm consisting of atypical polygonal cells forming irregular channels and dissecting dermal collagen (H\&E, original magnifications $\times 40$ and $\times 200$ ). for cytokeratin, CD10, CD68, human herpesvirus 8, CD34, and Melan A, thus confirming the endothelial origin of the tumor cells and the diagnosis of cutaneous angiosarcoma (CAS). The patient was treated with extended surgical excision and radiation therapy. No recurrence or metastasis was found throughout 2 years of follow-up.

Angiosarcoma is a highly aggressive malignant neoplasm derived from vascular endothelial cells, most
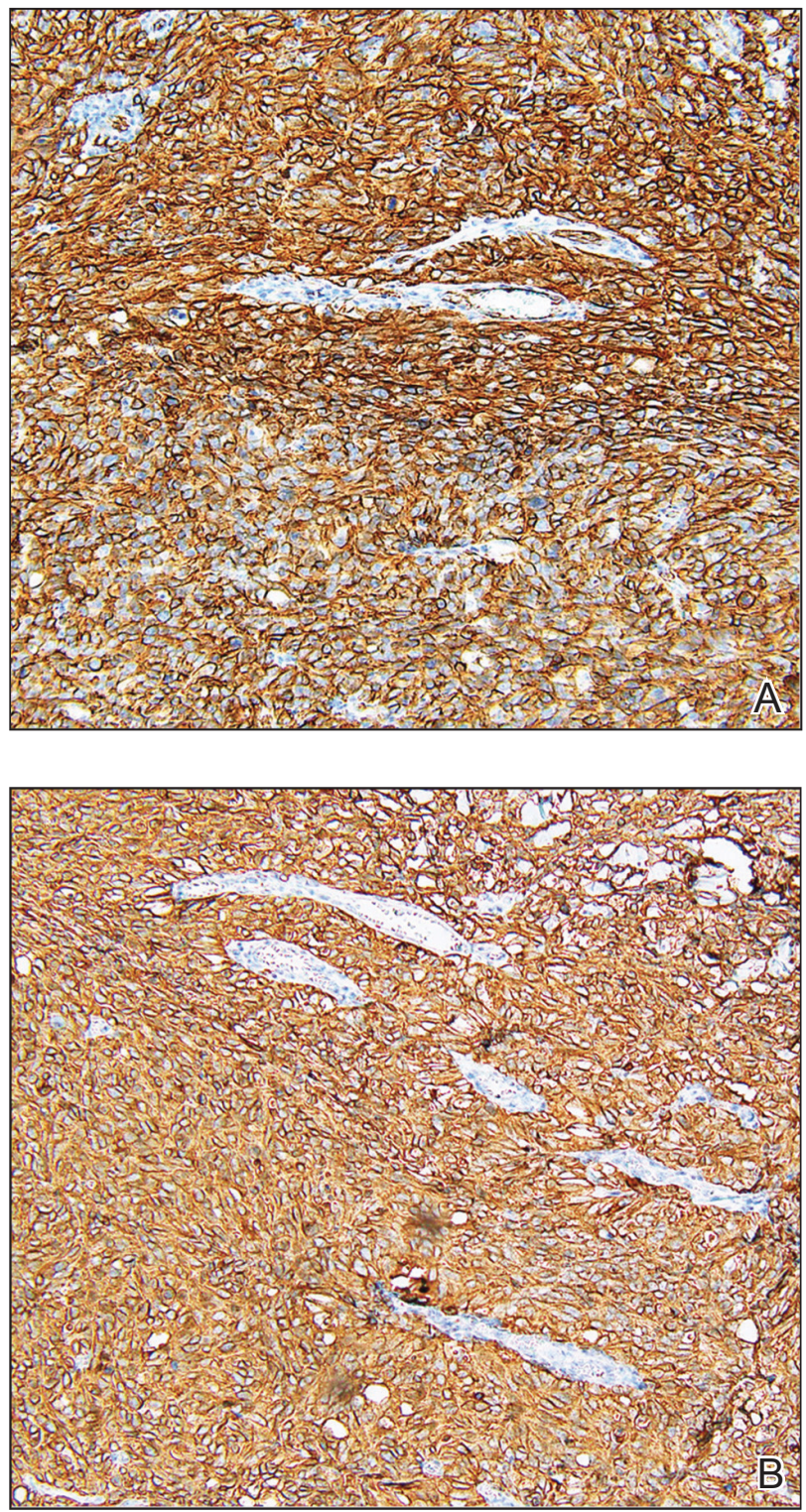

FIGURE 2. A and B, The endothelial origin was confirmed by immunohistochemistry for CD31 and D2-40, respectively (original magnifications $\times 100$ and $\times 100$ ) 
commonly involving the skin and superficial soft tissue. Angiosarcoma can be subdivided into CAS and visceral angiosarcoma according to the primary site of the tumor. ${ }^{1}$ Accurate and timely diagnosis of CAS is paramount due to its poor prognostic outcomes despite aggressive treatments. Clinically, CAS most frequently presents asymptomatically as an enlarging purple-red or bruiselike lesion with poorly defined margins. Cutaneous angiosarcoma often is misdiagnosed as an ecchymosis or hematoma due to its initial subtle presentation. It also may resemble eczema, hemangioma, and cellulitis; advanced lesions can mimic epithelial or mesenchymal neoplasms, including squamous cell carcinoma, keratoacanthoma, basal cell carcinoma, atypical fibroxanthoma (AFX), and malignant melanoma. ${ }^{2}$ Our patient lacked the classic clinical presentation of a hematomalike lesion and characteristic histologic features of anastomosing vascular structures with abundant extravasated erythrocytes at low magnification. However, the presence of erythrocytes in vascular channels along with CD31 and D2-40 immunoreactivity confirmed its vascular origin.

The prognosis of CAS is poor even with localized lesions. Age is a substantial prognostic factor, as a near $50 \%$ reduction of overall survival rate has been observed in patients older than 50 years. $^{3}$ Other reported poor predictors for prognosis include male sex, the presence of cardiovascular diseases, location on the scalp, history of smoking, tumor size larger than $5 \mathrm{~cm}$, and the presence of satellite lesions. Distant metastases are common, primarily affecting the lungs but also the bones and liver. ${ }^{4}$

Radical resection with a negative margin is considered the first-line treatment of choice. Although there is a paucity of studies assessing the specific width of surgical margins, application of no less than a $3-\mathrm{cm}$ peripheral margin as well as a clear deep margin is recommended. ${ }^{5}$ Adjuvant radiation therapy also is essential to prevent local recurrence. Patients receiving combination therapy have a superior overall survival rate when compared to those undergoing surgery or radiation therapy alone. ${ }^{4}$

Cutaneous follicle center lymphoma also may present as 1 or more localized erythematous papules, plaques, and/or nodules, commonly arising on the scalp/forehead or trunk of middle-aged men. Despite being a low-grade lymphoma with a favorable prognosis, it may have a relatively fast growth and locally aggressive course if left untreated. The distinguishing histologic feature is a dense proliferation of neoplastic infiltrates in the dermis, which is separated from the epidermis by the grenz zone. ${ }^{6}$

The clinical presentation of cutaneous metastatic carcinomas varies greatly, with 1 or multiple localized or widespread lesions commonly involving the abdominal wall, scalp, and face. The lesions also may mimic benign dermatologic conditions, thus potentially resulting in erroneous clinical diagnosis and delayed therapy of the primary malignancy. Obtaining clinical history is crucial; however, a precise diagnosis may require histologic examination.?

Atypical fibroxanthoma is a rare superficial cutaneous sarcoma that typically occurs on the head and neck in sun-damaged elderly individuals. Clinically, AFX presents as well-circumscribed red or pink nodules or plaques with or without ulceration, crust, or scale. ${ }^{8}$ Atypical fibroxanthoma lesions usually are small, with a median diameter of $1 \mathrm{~cm}$, while those greater than $2 \mathrm{~cm}$ reportedly account for less than $5 \%$ of cases. ${ }^{9}$ Atypical fibroxanthoma typically grows rapidly with no pain or discomfort. Histologically, AFX is characterized by a wellcircumscribed dermal nodule consisting of pleomorphic spindle cells and multinucleated giant cells that can stain positively for CD10 and procollagen $1 .{ }^{10}$

Cutaneous pseudolymphoma is a benign inflammatory response process that stimulates polyclonal T- or B-cell lymphoproliferation. The clinical presentation may appear as localized or disseminated fleshcolored or red papules, infiltrated plaques, and nodules. ${ }^{11}$ Histopathology will show mixtures of $\mathrm{B}$ and $\mathrm{T}$ cells along with dendritic cells and macrophages, but irregular vascular structure and dissecting dermal collagen are not involved.

We present an unusual case of CAS with multiple pink nodules on the scalp. Early biopsy of these lesions is important to reach a correct diagnosis and to initiate appropriate treatment.

\section{REFERENCES}

1. Ishida Y, Otsuka A, Kabashima K. Cutaneous angiosarcoma: update on biology and latest treatment. Curr Opin Oncol. 2018;30:107-112.

2. Dossett LA, Harrington M, Cruse CW, et al. Cutaneous angiosarcoma. Curr Probl Cancer. 2015;39:258-263.

3. Albores-Saavedra J, Schwartz AM, Henson DE, et al. Cutaneous angiosarcoma. analysis of 434 cases from the surveillance, epidemiology, and end results program, 1973-2007. Ann Diagn Pathol. 2011;15:93-97.

4. Guadagnolo BA, Zagars GK, Araujo D, et al. Outcomes after definitive treatment for cutaneous angiosarcoma of the face and scalp. Head Neck. 2011;33:661-667.

5. Lindford A, Böhling T, Vaalavirta L, et al. Surgical management of radiation-associated cutaneous breast angiosarcoma. J Plast Reconstr Aesthet Surg. 2011;64:1036-1042.

6. Costa EPW, Lu.0cena BD, Amin GA, et al. Primary cutaneous follicle center lymphoma. An Bras Dermatol. 2017;92:701-703.

7. Menon AR, Thomas AS, Suresh N, et al. Cutaneous metastasis: an unusual presenting feature of urologic malignancies. Urol Ann. 2016;8:377-380.

8. Iorizzo LJ 3rd, Brown MD. Atypical fibroxanthoma: a review of the literature. Dermatol Surg. 2011;37:146-157.

9. Kolb L, Schmieder GJ. Atypical fibroxanthoma. StatPearls. StatPearls Publishing; 2020

10. Sarac E, Yuksel M, Turkmen IC, et al. Case for diagnosis. atypical fibroxanthoma. An Bras Dermatol. 2019;94:239-241.

11. Miguel D, Peckruhn M, Elsner P. Treatment of cutaneous pseudolymphoma: a systematic review. Acta Derm Venereol. 2018;98:310-317. 